\title{
UNA PROPUESTA MULTIVARIANTE PARA MEDIR EL DESARROLLO HUMANO
}

\author{
Doris Gomez Ticerán ${ }^{1}$
}

\begin{abstract}
Un fenómeno tan complejo como el desarrollo humano, debería ser analizado holísticamente, por lo que se propone el uso de métodos estadísticos multivariantes (Anderson, 1984; Morrison, 1976; Bartholomew, 1987; Seber, 1984; Gómez, 1997; entre otros) para encontrar una variable combinada no observable denöminado Factor Desarrollo Humano (Morrison, 1976); - como alternativa - al HDI propuesto por primera vez por el Programa de las Naciones Unidas para el Desarrollo en el año 1990.

En el contexto descrito, el objetivo del presente trabajo es describir de manera sencilla el Perfil de Desarrollo Humano alcanzado por un conjunto de países mediante el Análisis Factorial (Anderson, 1984; Morrison, 1976; Bartholomew, 1987, Gómez, 1997, entre otros) y establecer una escala comparativa de desarrollo entre ellos.
\end{abstract}

\section{INTRODUCCIÓN}

El concepto tradicional del crecimiento económico se restringe a la expansión de la base material de la economía, sin embargo, el objetivo final del desarrollo económico debe ser la satisfacción de las necesidades humanas, es decir el desarrollo humano. Desde esta perspectiva es importante el crecimiento económico desde que la abundancia de productos sirva para enriquecer la vida de todas las personas y no sirva solamente para el bienestar de unos pocos en detrimento de la mayoría.

El enfoque de "oportunidades vitales" propuesto por Ralph Dahrendorf y el enfoque de Amartya Sen sobre "derechos y capacidades",

\footnotetext{
${ }^{1}$ Universidad Nacional Mayor de San Marcos. Facultad de Ciencias Matemáticas.
} 
han motivado la concepción de un nuevo enfoque de "desarrollo humano sostenible", propuesto por el Programa de las Naciones Unidas (PNUD).

La concepción sobre desarrollo humano que promueve el Programa de las Naciones Unidas (PNUD), fue lanzada en 1990. Este programa ha definido el desarrollo humano como un "proceso de ampliación de oportunidades de las personas", a quiénes considera como centro y sujetos del proceso de desarrollo, con la finalidad de que puedan tener una vida prolongada y saludable, con acceso a niveles de educación e ingresos acordes con la dignidad humana.

El Programa de las Naciones Unidas para el Desarrollo (PNUD) cuantifica el progreso de los pueblos mediante la construcción de un índice global de desenvolvimiento humano denominado Indice de Desarrollo Humano (HDI), con el fin de captar en un solo índice compuesto los logros del desarrollo humano.

El HDI es un instrumento que mide el adelanto medio de la capacidad humana básica de un país, respecto de tres dimensiones esenciales del desarrollo humano. Esperanza de vida, que refleja una existencia larga y saludable; nivel educacional, que resume los conocimientos adquiridos; el ingreso per-cápita, que indica la capacidad de acceso a los recursos para vivir dignamente. Es decir, se combinan indicadores de salud, educación y economía, y por definición es una medida estandarizada $(0 \leq H D I \leq 1)$ que representa la habilidad que tienen los paises para vivir una vida saludable y larga, participar de la cultura y tener recursos para una vida de calidad. Países con $H D I$ superior a 0.8 son clasificados como de desarrollo humano alto, con $H D I$ entre 0.795 y 0.5 con desarrollo humano medio y los paises con $H D I$ por debajo de 0.5 son clasificados con desarrollo humano bajo.

Cabe señalar que un fenómeno tan complejo como el desarrollo humano, debería ser analizado holísticamente, para lo cual me permito proponer el uso de métodos estadísticos multivariantes, que se prueba pueden competir con la metodología de las Naciones Unidas.

En el contexto descrito, el objetivo del presente trabajo es describir de manera sencilla el Perfil de Desarrollo Humano alcanzado por un conjunto de países mediante el Análisis Factorial (Anderson, 1984; Morrison, 1976; Bartholomew, 1987, Gómez, 1997, entre otros). Con este fin se seleccionaron variables que combinadas adecuadamente permiten conseguir el objetivo propuesto. 


\section{METODOLOGIA}

\section{MODELO}

Por la dificultad que tiene el análisis simultáneo de las variables involucradas en el concepto Desarrollo Humano, alcanzado por los diversos paises considerados en éste análisis, se utilizó el Análisis de Factores por el Método de Componentes Principales (Mardia, 1979; Anderson, 1984; Bartholomew, 1987)para obtener un número menor de variables latentes no observables, denominados Factores Comunes, que luego son usados como medida del grado de desarrollo de los países considerados. A continuación se presenta una breve descripción del modelo.

El Análisis de Factores es un modelo estadístico que permite explicar la estructura de las correlaciones que existe entre un conjunto grande de variables observadas, $X_{i}, i=1, \ldots, p$, mediante un número pequeño de variables no observadas, latentes, denominados factores comunes, $f_{j}, j=1, \ldots, m$; que son de interés para el investigador, como en la situación descrita. Es decir, pretendemos construir un factor latente no observable denominado Desarrollo Humano, a partir de un conjunto grande de $p$ variables observables directamente.

La condición clave para hacer el Análisis Factorial es la suposición de la existencia de pocas variables subyacentes no medibles, denominados factores, que hacen posible la existencia de las correlaciones o asociaciones entre todas las variables observadas.

En el modelo factorial, cada una de las $p$ variables es combinación lineal de los $m$ - factores no observables o factores comunes y de una única variable denominado factor específico. Los dos tipos de factores, los comunes y los específicos son latentes y, no son observables directamente por el investigador.

Los $m$ factores comunes, contribuyen en la formación de las covarianzas de las variables observables, mientras que el factor específico contribuye sólo en la formación de la varianza de cada una de las variables.

Formalmente el modelo es el siguiente:

- Sea $\left(x_{1}, \ldots, x_{p}\right)^{\prime}$ el vector aleatorio con vector de medias $\vec{u}=$ $\left(u_{1}, \ldots, u_{p}\right)^{\prime}$ y matriz de covarianzas $\Sigma$.

- El Modelo Factorial para el vector $\left(x_{i}, \ldots, x_{p}\right)$ de variables originales toma la forma:

$$
\vec{x}=\Lambda \vec{f}+\vec{e}
$$


donde :

$\vec{f}=\left(f_{j}\right)$ : Vector aleatorio. Sus elementos son denominados factores comunes;

$\vec{e}=\left(e_{j}\right)$ : Vector aleatorio. Sus elementos son denominados factores específicos;

$\Delta=\left(\lambda_{i j}\right)$ : matriz de ponderaciones de los factores comunes refleja la importancia del $j$-ésimo factor común en la composición de la $i$-ésima variable;

$\mathrm{m}$ : número de factores latentes no observables $(<p)$

p: número de variables originales, con los supuestos:

$$
\begin{array}{lll}
E(\vec{f})=0 & \operatorname{Var}(\vec{f})=I & \text { matriz identidad } \\
E(\vec{e})=0 & \operatorname{Var}(\vec{e})=\Psi & \text { matriz diagonal } \\
\operatorname{Cov}(\vec{f}, \vec{e})=0 &
\end{array}
$$

donde todos los factores (comunes y no comunes) están no correlacionados; los que deben permitir descomponer la estructura de la matriz de covarianzas según el Modelo Factorial, es decir de la siguiente manera:

$$
\Sigma=\Lambda \Lambda^{\prime}+\Psi
$$

Según (3) la varianza de la $i$-ésima variable se descompone en dos partes, la comunalidad y la especificidad:

donde:

$$
\operatorname{Var}\left(X_{i}\right)=\Sigma \lambda_{i j}^{2}+\Psi_{i i}
$$

- Comunalidad $\left(\Sigma \lambda_{i j}^{2}\right)$ : Es la parte de la varianza de la variable, $X_{i}$, que es explicada por los factores comunes, $f_{j}$.

En particular, $\lambda_{i j}^{2}=$ Correlación $\left(X_{i}, f_{j}\right)$ es el tamaño de la variabilidad de la $i$-ésima variable, $X_{i}$, que depende del $j$-ésimo factor, $f_{j}$.

- Especificidad: $\Psi_{i i}$ es la parte de la varianza de la variable, $X_{i}$, que es explicada por el factor específico. Támbién denominada varianza específica. 
Dado una muestra, el problema básico es decidir si la matriz de covarianzas puede expresarse en la forma (3), mediante un conjunto de $m \leq p$ factores, y estimar todos los parámetros del modelo,(1)usando de manera conveniente los supuestos (2).

- La contribución total del j-ésimo factor a la varianza total de las $p$ variables es:

$$
V_{j}=\Sigma \lambda_{i j}^{2}=\lambda_{(j)}^{\prime} \lambda_{(j)} \text { donde } \lambda_{(j)}: j \text { - ésima columna de } \Lambda \text {. }
$$

- La contribución total de todos los factores a la varianza total de todas las variables es:

$$
V=\Sigma V_{j}=\Sigma \Sigma \lambda_{i j}^{2}
$$

- $V_{j} / V$ : Porcentaje de la varianza total de todas las variables, que es explicado por el $j$-ésimo factor

$$
\text { Varianza Total }=\operatorname{Tr}(\Sigma)=\Sigma \sigma_{i i}=\Sigma V_{j}+\Sigma \Psi_{i i}=\Sigma \Sigma \lambda_{i j}^{2}+\Sigma \Psi_{i i}
$$

Cabe resaltar que $\vec{f}$ y $\vec{e}$ no son únicos, por lo que se hace necesario hacer rotaciones de los factores, para lo cual se introduce la restricción: $\Lambda^{\prime} \Psi^{-1} \Lambda=D$, donde $D$ es una matriz diagonal $\left(\operatorname{diag}\left(\sigma_{i i}, \ldots, \sigma_{p p}\right)\right)$.

\section{DESCRIPCIÓN DE LOS DATOS}

La fuente de información fue: Human Development Report, 1998 (UNDP) y las variables escogidas fueron:

$X_{1}$ : Tasa bruta de mortalidad , 1997

$X_{2}$ : Tasa bruta de natalidad, 1997

$X_{3}$ : Tasa global de fecundidad, 1997

$X_{4}$ : Tasa anual de crecimiento de la población urbana(\%), 1997

$X_{5}$ : Tasa de mortalidad infantil antes del año (\%), 1997

$X_{6}$ : Tasa bruta de escolaridad enseñanza primaria, 1997

$X_{7}$ : Producto nacional bruto per cápita (dólares), 1997

$X_{8}$ : Población inmunizada contra la polio (\%), 1995-1997

$X_{9}$ : Recién nacidos(\%) con bajo peso, 1997

$X_{10}$ : Tasa bruta de escolaridad primaria (\%), 1990-1996

$X_{11}$ : Tasa de alfabetización de adultos (\%), 1997

$X_{12}$ : Producto bruto interno per cápita (PPA en dólares), 1997

$X_{13}$ : Esperanza de vida al nacer(años), 1997 
A pesar de ser muy importantes, algunas otras variables como por ejemplo: población con acceso a servicios de agua y población con acceso a servicios de salud, no fueron considerados en el presente análisis por falta de información para algunos países tales como Argentina, Perú y Cuba.

Cabe observar que estamos considerando en la muestra solamente países no industrializados, para de alguna manera, controlar la distorsión debida a la situación de ser y no ser industrializado.

\section{RESULTADOS}

El análisis se ha llevado a cabo con la información contenida en la matriz de correlaciones, la que mostró altas correlaciones entre la mayoría de variables involucradas.

En la Tabla $\mathrm{N}^{\circ} 1$ se tienen los porcentajes de explicación de los dos primeros factores. Así, el primer factor explica el $55.4 \%$ y el segundo factor el $15.19 \%$ de la variación total contenida en el problema original. Los dos primeros factores explican el $70.59 \%$ del problema en estudio y llevando en consideración estos resultados se optó por considerar dos factores para el estudio.

Luego, se escogió la rotación varimax (Bartholomew, 1981) porque facilita la interpretación de las ponderaciones de los factores.

Tabla $N^{\circ} 1$ - Autovalores de la Matriz de Correlaciones

\begin{tabular}{|c|c|c|c|}
\hline \multirow[t]{2}{*}{ Factores } & \multirow[t]{2}{*}{ Autovalor } & \multicolumn{2}{|c|}{ Proporción } \\
\hline & & Simple & Acumulada \\
\hline 1 & 6.65 & 55.40 & 55.40 \\
\hline 2 & 1.82 & 15.19 & 70.59 \\
\hline Otros & 3.53 & 29.41 & 100.00 \\
\hline Total & 12.00 & 100.00 & \\
\hline
\end{tabular}

En la Tabla $\mathrm{N}^{\circ} 2$ se tienen las cargas factoriales y la descomposición de las varianzas de cada variable en su comunalidad y su especificidad, después de la rotación. 
Tabla N² - Cargas Factoriales de los Factores y las Comunalidades después de la Rotación

\begin{tabular}{|c|c|c|c|c|c|c|}
\hline Variables & $\begin{array}{c}\text { Cargas } \\
f_{1}\end{array}$ & $\begin{array}{l}\text { actoriales } \\
f_{2}\end{array}$ & $\begin{array}{l}\mathrm{Co} \\
f_{1}\end{array}$ & $\begin{array}{c}\text { nunalic } \\
f_{2}\end{array}$ & $\begin{array}{l}\text { ad } \\
\text { total }\end{array}$ & $\begin{array}{c}\text { Especificidad } \\
\Psi_{i}\end{array}$ \\
\hline Mortalidad & -0.677 & -0.154 & 0.458 & 0.024 & 0.482 & 0.518 \\
\hline Natalidad & -0.518 & -0.673 & 0.268 & 0.453 & 0.721 & 0.279 \\
\hline Fecundidad & -0.585 & -0.568 & 0.342 & 0.323 & 0.665 & 0.335 \\
\hline Crec.Pob. & -0.378 & -0.736 & 0.143 & 0.542 & 0.685 & 0.315 \\
\hline Mort.Infan. & -0.657 & -0.623 & 0.432 & 0.388 & 0.820 & 0.180 \\
\hline Escolaridad & 0.785 & -0.061 & 0.616 & 0.004 & 0.620 & 0.380 \\
\hline Pnb & 0.043 & 0.894 & 0.002 & 0.799 & 0.801 & 0.199 \\
\hline Inm.polio & 0.566 & 0.333 & 0.320 & 0.111 & 0.431 & 0.569 \\
\hline Bajo peso & -0.499 & -0.380 & 0.249 & 0.144 & 0.393 & 0.607 \\
\hline t.b.escolar & 0.918 & 0.097 & 0.843 & 0.009 & 0.852 & 0.148 \\
\hline t.alfabet. & 0.721 & 0.513 & 0.520 & $0.263^{*}$ & 0.783 & 0.217 \\
\hline PBI per cáp. & 0.060 & 0.915 & 0.004 & 0.837 & 0.841 & 0.159 \\
\hline Esperanza & 0.702 & 0.652 & 0.493 & 0.425 & 0.918 & 0.082 \\
\hline
\end{tabular}

Las variables mejor caracterizadas por los factores $f_{1}$ y $f_{2}$ son la esperanza de vida al nacer, la tasa bruta de escolaridad, el producto bruto interno, la tasa de mortalidad infantil, el producto nacional bruto, la tasa de alfabetización, la tasa de natalidad, la tasa de fecundidad, el crecimiento poblacional, la tasa de escolaridad. Estas son las variables que permiten dar nombre a los factores.

Por otro lado, se sabe que en toda situación donde se trabaja con métodos factoriales, de los que forma parte el Análisis Factorial, la principal dificultad es dar nombre a los factores o variables latentes que en el presente trabajo están representados por los factores $f_{1}$ y $f_{2}$.

Observando las ponderaciones del factor $f_{1}$ podemos afirmar que está negativamente correlacionado con indicadores demográficos y de nutrición como son: tasa de mortalidad, tasa de natalidad, tasa de fecundidad tasa de crecimiento poblacional urbano, tasa de mortalidad antes del año, porcentaje de recién nacidos con bajo peso. Por otro lado está positivamente correlacionado con los indicadores sociales básicos: tasa bruta de escolaridad de enseñanza primaria, población inmunizada contra el polio, tasa bruta de escolaridad primaria del sexo femenino, tasa de alfabetización de adultos y esperanza de vida al nacer. Por la alta correlación observada entre el factor $f_{1}$ y las variables descritas podemos afirmar que este factor representa condiciones de desarrollo 
humano, dado que este factor es una combinación de indicadores demográficos, indicadores de salud, indicadores educativos y de nutrición.

Por otro lado, se observa que el factor $f_{2}$ tiene fuerte correlación positiva con el producto bruto interno y la renta per cápita, representado un factor de desarrollo económico.

Así por ejemplo, a menor mortalidad mayor desarrollo humano, a mayor tasa de escolaridad primaria mayor desarrollo humano; mientras que a mayor producto bruto interno mayor desarrollo económico.

En conjunto, los factores $f_{1}$ y $f_{2}$ estarían representando el perfil de desarrollo humano y desarrollo económico de los países considerados en el presente trabajo.

Por otro lado, sabemos que los factores $f_{1}$ y $f_{2}$ no están correlacionados y esto nos confirma que el desarrollo humano y el desarrollo económico son factores que en la vida real no se correlacionan y es un asunto en el que deberían trabajar las respectivas organizaciones responsables a fin de que en el mediano plazo pueda revertirse esta situación.

La Tabla $N^{\circ} 3$ muestra para cada uno de los países considerados en el estudio, los valores de los dos factores construidos(desarrollo humano y desarrollo económico) además de la clasificación proporcionada por el Human Development Report (1999).

Sabemos que los factores $f_{1}$ y $f_{2}$ son variables (no observadas) con media igual a cero y desviación estandar igual a uno. Esto significa que valores cercanos a cero para los dos factores indica un grado de desarrollo humano y económico medio. Una situación de atraso relativo se manifiesta a través de valores negativos en los dos factores. Valores positivos en los dos factores indican una situación de mejores condiciones de desarrollo humano y económico.

A partir de los resultados de la Tabla $\mathrm{N}^{\circ} 3$ algunos de los países de desarrollo humano alto son: Uruguay, Argentina, Trinidad y Tobago, Méjico, Panamá con valores positivos y altos en $f_{1}$.

Tabla $N^{\circ} 3$

\begin{tabular}{|lcccc|}
\hline PAIS & $\begin{array}{c}\text { IDH } \\
(*)\end{array}$ & $\begin{array}{c}\text { ORDEN } \\
\text { ASIGNADO }\end{array}$ & FACTOR 1 & ORDEN \\
\hline Islandia & .9190 & 1 & DESARROLLO & ALTO \\
Irlanda & .9000 & 3 & 2.28027 & 1 \\
\end{tabular}


Doris Gomez Ticerán

\begin{tabular}{|c|c|c|c|c|}
\hline PAIS & $\begin{array}{l}\text { IDH } \\
(*)\end{array}$ & $\begin{array}{c}\text { ORDEN } \\
\text { ASIGNADO }\end{array}$ & FACTOR 1 & ORDEN \\
\hline Portugal & .8580 & 6 & 1.91042 & 3 \\
\hline Finlandia & .9130 & 2 & 1.29484 & 4 \\
\hline Chile & .8440 & 9 & .76799 & 5 \\
\hline Malta & .8500 & 8 & .76491 & 6 \\
\hline Barbado & .8570 & 7 & .76080 & 7 \\
\hline Argentina & .8270 & 11 & .74598 & 8 \\
\hline Mexico & .7860 & 17 & .74554 & 9 \\
\hline Singapur & .8880 & 4 & .73150 & 10 \\
\hline Kuwait & .8330 & 10 & .72664 & 11 \\
\hline Uruguay & .8260 & 12 & .72504 & 12 \\
\hline Costa Rica & .8010 & 13 & .71544 & 13 \\
\hline Venezuela & .7920 & 15 & .70168 & 14 \\
\hline Colombia & .7680 & 20 & .68361 & 15 \\
\hline Panamá & .7910 & 16 & .66493 & 16 \\
\hline & & & DESARROLLO & MEDIO \\
\hline Israel & .8830 & 5 & .64145 & 17 \\
\hline Cuba & .7650 & 21 & .59981 & 18 \\
\hline Dominica & .7760 & 18 & .50641 & 19 \\
\hline Trin.y Tobago & .7970 & 14 & .49625 & 20 \\
\hline Fiji & .7630 & 23 & .39262 & 21 \\
\hline Arabias & .7400 & 28 & .39154 & 22 \\
\hline Paraguay & .7300 & 32 & .37161 & 23 \\
\hline Albania & .6990 & 38 & .32193 & 24 \\
\hline Brasil & .7390 & 30 & .31884 & 25 \\
\hline PERU & .7390 & 29 & .27640 & 26 \\
\hline Armenia & .7280 & 34 & .27549 & 27 \\
\hline Ecuador & .7470 & 26 & .25339 & 28 \\
\hline Jamaica & .7340 & 31 & .09229 & 29 \\
\hline turquía & .7280 & 33 & .05103 & 30 \\
\hline El Salvador & .6740 & 40 & .03811 & 31 \\
\hline China & .7010 & 36 & .03754 & 32 \\
\hline Mauricio & .7640 & 22 & .01737 & 33 \\
\hline & & & DESARROLLO & BAJO \\
\hline Malasia & .7680 & 19 & -.10837 & 34 \\
\hline Filipinas & .7400 & 27 & -.14272 & 35 \\
\hline Líbano & .7490 & 25 & -.14363 & 36 \\
\hline Egipto & .6160 & 46 & -.18331 & 37 \\
\hline Jordania & .7150 & 35 & -.18499 & 38 \\
\hline Mongolia & .6180 & 45 & -.55057 & 39 \\
\hline
\end{tabular}


UNA PROPUESTA MULTIVARIANTE PARA MEDIR ...

\begin{tabular}{|lcccc|}
\hline PAIS & $\begin{array}{c}\text { IDH } \\
(*)\end{array}$ & $\begin{array}{c}\text { ORDEN } \\
\text { ASIGNADO }\end{array}$ & FACTOR 1 & ORDEN \\
\hline Marruecos & .5820 & 49 & -.59305 & 40 \\
Bangladesh & .4400 & 54 & -.61516 & 41 \\
Bolivia & .6520 & 42 & -.61549 & 42 \\
Mozambique & .3410 & 56 & -.64004 & 44 \\
Kenya & .5190 & 52 & -.75765 & 43 \\
Lituania & .7610 & 24 & -.84760 & 45 \\
Argelia & .6650 & 41 & -.93973 & 46 \\
India & .5450 & 51 & -1.33305 & 47 \\
Indonesia & .6820 & 39 & -1.34558 & 48 \\
Honduras & .6410 & 43 & -1.40003 & 49 \\
Guyana & .7010 & 37 & -1.55855 & 50 \\
Pakistan & .5080 & 53 & -1.57457 & 51 \\
Etiopia & .2890 & 57 & -1.62615 & 52 \\
Irak & .5860 & 48 & -1.80094 & 53 \\
Haiti & .4300 & 55 & -1.93144 & 54 \\
Guatemala & .6240 & 44 & -2.05492 & 55 \\
Birmania & .5800 & 50 & -2.06986 & 56 \\
Nicaragua & .6160 & 47 & -2.97014 & 57 \\
\hline
\end{tabular}

* Fuente: Human Development Report (1999), UNDP.

Algunos países con desarrollo medio: Cuba, Brasil, Perú y Ecuador entre otros. Países con desarrollo bajo: Bolivia, Honduras y Nicaragua entre otros, pues tienen los más bajos valores en el factor $f_{1}$.

\section{CONCLUSIONES}

En términos generales, en lo referente a los países considerados el análisis muestra que el factor Desarrollo Humano sería una alternativa al HDI construido por el Programa de las Naciones Unidas.

Entre otros países, el Perú aparece como un país en la media de desarrollo.

En términos generales, los resultados a los que se llega en la presente investigación son coherentes con el $H D I$, a pesar de ser otra metodología de análisis y de no haber considerado muchas variables de interés para la explicación del fenómeno estudiado. 


\section{BIBLIOGRAFÍA}

[1] E.B. Andersen, Latent structure analysis., Scand. J. Stat., 1,(1992), 115-127.

[2] T.W. Anderson, Introduction to Multivariate Statistical Analysis.,Wiley, New York, (1984).

[3] M.S. Bartlett, The effects of standardisation on an approximation in factor analysis.,Biometrika, 38,(1951),pp.337-344.

[4] D. Bartholomew, Posterior Analysis of the factor model., Br. J. Math. Stat. Psychol., 24,(1991), 93-99.

[5] D. Bartholomew, Latent Variable Model and Factor Analysis. Charles Griffin and Company Limited.

[6] D. Gómez, Una Aplicación del Análisis Multivariante. Viernes Culturales. Boletín Interno de la Escuela Académico Profesional de Matemáticas. UNMSM. Octubre, 1997.

[7] G.A.Seber, Multivariate Observations. Wiley Series in Probablity and Mathematical Statistics.(1984). 